\title{
Some Integral Type Fixed Point Theorems in Dislocated Metric Space
}

\author{
Dinesh Panthi' ${ }^{1}$ Panda Sumati Kumari ${ }^{2}$ \\ ${ }^{1}$ Department of Mathematics, Valmeeki Campus, Nepal Sanskrit University, Kathmandu, Nepal \\ ${ }^{2}$ Department of Mathematics, National Institute of Technology, Andhra Pradesh, Tadepalligudem, India \\ Email: panthid06@gmail.com
}

Received 6 April 2016; accepted 6 June 2016; published 9 June 2016

Copyright (C) 2016 by authors and Scientific Research Publishing Inc.

This work is licensed under the Creative Commons Attribution International License (CC BY).

http://creativecommons.org/licenses/by/4.0/

(c) (i) Open Access

\begin{abstract}
In this article, we establish a common fixed point theorem satisfying integral type contractive condition for two pairs of weakly compatible mappings with E. A. property and also generalize Theorem (2) of B.E. Rhoades [1] in dislocated metric space.
\end{abstract}

\section{Keywords}

Dislocated Metric, Weakly Compatible Maps, Common Fixed Point

\section{Introduction}

In 1986, S. G. Matthews [2] introduced some concepts of metric domains in the context of domain theory. In 2000, P. Hitzler and A.K. Seda [3] introduced the concept of dislocated topology where the initiation of dislocated metric space was appeared. Since then, many authors have established fixed point theorems in dislocated metric space. In the literature, one can find many interesting recent articles in the field of dislocated metric space (see for examples [4]-[10]).

The study of fixed point theorems of mappings satisfying contractive conditions of integral type has been a very interesting field of research activity after the establishment of a theorem by A. Branciari [11]. The purpose of this article is to establish a common fixed point theorem for two pairs weakly compatible mappings with E. A. property and to generalize a result of B.E. Rhoades [1] in dislocated metric space.

\section{Preliminaries}

We start with the following definitions, lemmas and theorems.

Definition 1 [3] Let $X$ be a non empty set and let $d: X \times X \rightarrow[0, \infty)$ be a function satisfying the following 
conditions:

1. $d(x, y)=d(y, x)$.

2. $d(x, y)=d(y, x)=0$ implies $x=y$.

3. $d(x, y) \leq d(x, z)+d(z, y)$ for all $x, y, z \in X$.

Then $d$ is called dislocated metric (or d-metric) on $X$ and the pair $(X, d)$ is called the dislocated metric space (or d-metric space).

Definition 2 [3] A sequence $\left\{x_{n}\right\}$ in a d-metric space $(X, d)$ is called a Cauchy sequence if for given $\epsilon>0$, there corresponds $n_{0} \in N$ such that for all $m, n \geq n_{0}$, we have $d\left(x_{m}, x_{n}\right)<\epsilon$.

Definition 3 [3] A sequence in d-metric space converges with respect to $d$ (or in $d$ ) if there exists $x \in X$ such that $d\left(x_{n}, x\right) \rightarrow 0$ as $n \rightarrow \infty$.

Definition 4 [3] A d-metric space $(X, d)$ is called complete if every Cauchy sequence in it is convergent with respect to $d$.

Lemma 1 [3] Limits in a d-metric space are unique.

Definition 5 Let $A$ and $S$ be two self mappings on a set $X$. If $A x=S x$ for some $x \in X$, then $x$ is called coincidence point of $A$ and $S$.

Definition 6 [12] Let $A$ and $S$ be mappings from a metric space $(X, d)$ into itself. Then, $A$ and $S$ are said to be weakly compatible if they commute at their coincident point; that is, Ax $=S x$ for some $x \in X$ implies $A S x=S A x$.

Definition 7 [13] Let $A$ and $S$ be two self mappings defined on a metric space $(X, d)$. We say that the mappings $A$ and $S$ satisfy (E. A.) property if there exists a sequence $\left\{x_{n}\right\} \in X$ such that

$$
\lim _{n \rightarrow \infty} A x_{n}=\lim _{n \rightarrow \infty} S x_{n}=u
$$

for some $u \in X$.

\section{Main Results}

Now we establish a common fixed point theorem for two pairs of weakly compatible mappings using E. A. property.

Theorem 1 Let $(X, d)$ be a dislocated metric space. Let $A, B, S, T: X \rightarrow X$ satisfying the following conditions

$$
\begin{gathered}
A(X) \subseteq S(X) \quad \text { and } \quad B(X) \subseteq T(X) \\
\int_{0}^{d(A x, B y)} \phi(t) \mathrm{d} t \leq k \int_{0}^{M(x, y)} \phi(t) \mathrm{d} t, \quad k \in\left[0, \frac{1}{2}\right)
\end{gathered}
$$

where

$$
\phi: \mathbb{R}^{+} \rightarrow \mathbb{R}^{+}
$$

is a Lebesgue integrable mapping which is summable, non-negative and such that

$$
\begin{gathered}
\int_{0}^{\epsilon} \phi(t) \mathrm{d} t>0 \quad \text { for each } \epsilon>0 \\
M(x, y)=\max \{d(S y, A x), d(T x, S y), d(T x, A x), d(B y, S y), d(T x, B y)\}
\end{gathered}
$$

1. The pairs $(A, T)$ or $(B, S)$ satisfy E. A. property.

2. The pairs $(A, T)$ and $(B, S)$ are weakly compatible.

if $T(X)$ is closed then

1) the maps $\mathrm{A}$ and $\mathrm{T}$ have a coincidence point.

2 the maps $B$ and $S$ have a coincidence point.

3) the maps A, B, S and T have an unique common fixed point.

Proof. Assume that the pair $(A, T)$ satisfy E.A. property, so there exists a sequence $\left\{x_{n}\right\} \in X$ such that

$$
\lim _{n \rightarrow \infty} A x_{n}=\lim _{n \rightarrow \infty} T x_{n}=u
$$


for some $u \in X$. Since $A(X) \subseteq S(X)$, so there exists a sequence $\left\{y_{n}\right\} \in X$ such that $A x_{n}=S y_{n}$. Hence,

$$
\lim _{n \rightarrow \infty} A x_{n}=\lim _{n \rightarrow \infty} S y_{n}=u
$$

From condition (2) we have

$$
\int_{0}^{d\left(A x_{n}, B y_{n}\right)} \phi(t) \mathrm{d} t \leq k \int_{0}^{M\left(x_{n}, y_{n}\right)} \phi(t) \mathrm{d} t,
$$

where

$$
M\left(x_{n}, y_{n}\right)=\max \left\{d\left(S y_{n}, A x_{n}\right), d\left(T x_{n}, S y_{n}\right), d\left(T x_{n}, A x_{n}\right), d\left(B y_{n}, S y_{n}\right), d\left(T x_{n}, B y_{n}\right)\right\}
$$

Taking limit as $n \rightarrow \infty$ we get

$$
\lim _{n \rightarrow \infty} \int_{0}^{d\left(A x_{n}, B y_{n}\right)} \phi(t) \mathrm{d} t \leq k \lim _{n \rightarrow \infty} \int_{0}^{M\left(x_{n}, y_{n}\right)} \phi(t) \mathrm{d} t,
$$

Since

$$
\begin{gathered}
\lim _{n \rightarrow \infty} d\left(T x_{n}, S y_{n}\right)=\lim _{n \rightarrow \infty} d\left(T x_{n}, A x_{n}\right)=\lim _{n \rightarrow \infty} d\left(S y_{n}, A x_{n}\right)=0 \\
\lim _{n \rightarrow \infty} d\left(B y_{n}, S y_{n}\right)=\lim _{n \rightarrow \infty} d\left(T x_{n}, B y_{n}\right)=d\left(B y_{n}, u\right)
\end{gathered}
$$

Hence we have

$$
\int_{0}^{d\left(u, B y_{n}\right)} \phi(t) \mathrm{d} t \leq k \int_{0}^{d\left(u, B y_{n}\right)} \phi(t) \mathrm{d} t,
$$

which is a contradiction, since $k \in\left[0, \frac{1}{2}\right)$. Hence, $\lim _{n \rightarrow \infty} B y_{n}=u$. Now we have

$$
\lim _{n \rightarrow \infty} A x_{n}=\lim _{n \rightarrow \infty} T x_{n}=\lim _{n \rightarrow \infty} B y_{n}=\lim _{n \rightarrow \infty} S y_{n}=u
$$

Assume $T(X)$ is closed, then there exits $v \in X$ such that $T v=u$. We claim that $A v=u$. Now from condition (2)

$$
\int_{0}^{d\left(A v, B y_{n}\right)} \phi(t) \mathrm{d} t \leq k \int_{0}^{M\left(v, y_{n}\right)} \phi(t) \mathrm{d} t,
$$

where

$$
M\left(v, y_{n}\right)=\max \left\{d\left(S y_{n}, A v\right), d\left(T v, S y_{n}\right), d(T v, A v), d\left(B y_{n}, S y_{n}\right), d\left(T v, B y_{n}\right)\right\}
$$

Since

$$
\begin{gathered}
\lim _{n \rightarrow \infty} d\left(S y_{n}, A v\right)=d(u, A v) \\
\lim _{n \rightarrow \infty} d\left(T v, S y_{n}\right)=\lim _{n \rightarrow \infty} d\left(B y_{n}, S y_{n}\right)=\lim _{n \rightarrow \infty} d\left(T v, B y_{n}\right)=0
\end{gathered}
$$

So, taking limit as $n \rightarrow \infty$ in (9), We conclude that

$$
\int_{0}^{d(A v, u)} \phi(t) \mathrm{d} t \leq k \int_{0}^{d(u, A v)} \phi(t) \mathrm{d} t
$$

which is a contradiction. Hence $d(A v, u)=0 \Rightarrow A v=u$. Now we have

$$
A v=u=T v \text {. }
$$

This proves that $v$ is the coincidence point of $(A, T)$.

Again, since $A(X) \subseteq S(X)$ so there exists $w \in X$ such that

$$
A v=S w=u
$$

Now we claim that $B w=u$. From condition (2)

$$
\int_{0}^{d(u, B w)} \phi(t) \mathrm{d} t=\int_{0}^{d(A v, B w)} \phi(t) \mathrm{d} t \leq k \int_{0}^{M(v, w)} \phi(t) \mathrm{d} t,
$$


where

$$
\begin{aligned}
M(v, w) & =\max \{d(S w, A v), d(T v, S w), d(T v, A v), d(B w, S w), d(T v, B w)\} \\
& =\max \{d(u, u), d(u, u), d(u, u), d(B w, u), d(u, B w)\} \\
& =\max \{d(u, u), d(B w, u)\}
\end{aligned}
$$

Since

$$
d(u, u) \leq 2 d(u, B w)
$$

So if $\max \{d(u, u), d(B w, u)\}=d(u, u)$ or $d(B w, u)$ we get the contradiction, since

$$
\int_{0}^{d(u, B w)} \phi(t) \mathrm{d} t \leq 2 k \int_{0}^{d(u, B w)} \phi(t) \mathrm{d} t
$$

or

$$
\int_{0}^{d(u, B w)} \phi(t) \mathrm{d} t \leq k \int_{0}^{d(u, B w)} \phi(t) \mathrm{d} t
$$

Hence, $d(u, B w)=0 \Rightarrow B w=u$.

Therefore, $B w=u=S w$.

This represents that $w$ is the coincidence point of the maps $B$ and $S$.

Hence,

$$
u=B w=S w=T v=A v
$$

Since the pairs $(B, S)$ and $(A, T)$ are weakly compatible so,

$$
\begin{gathered}
B S w=S B w, \quad T A v=A T v \\
T u=T A v=A T v=A u \quad \text { and } \quad S u=S B w=B S w=B u
\end{gathered}
$$

We claim $B u=u$. From condition (2)

$$
\int_{0}^{d(u, B u)} \phi(t) \mathrm{d} t=\int_{0}^{d(A v, B u)} \phi(t) \mathrm{d} t \leq k \int_{0}^{M(v, u)} \phi(t) \mathrm{d} t
$$

where

$$
\begin{aligned}
M(v, u) & =\max \{d(S u, A v), d(T v, S u), d(T v, A v), d(B u, S u), d(T v, B u)\} \\
& =\max \{d(B u, u), d(u, B u), d(u, u), d(B u, B u), d(u, B u)\} \\
& =\max \{d(u, B u), d(u, u), d(B u, B u)\}
\end{aligned}
$$

Since

$$
d(u, u) \leq 2 d(u, B u) \text { and } d(B u, B u) \leq 2 d(u, B u)
$$

So if $\max \{d(u, B u), d(u, u), d(B u, B u)\}=d(u, B u)$ or $d(u, u)$ or $d(B u, B u)$ we get the contradiction. Since,

$$
\int_{0}^{d(u, B u)} \phi(t) \mathrm{d} t=\int_{0}^{d(A v, B u)} \phi(t) \mathrm{d} t \leq k \int_{0}^{M(v, u)} \phi(t) \mathrm{d} t \leq k \int_{0}^{d(u, B u)} \phi(t) \mathrm{d} t
$$

or

$$
\int_{0}^{d(u, B u)} \phi(t) \mathrm{d} t \leq 2 k \int_{0}^{d(u, B u)} \phi(t) \mathrm{d} t
$$

Hence, $d(u, B u)=0 \Rightarrow B u=u$.

Therefore, $u=B u=S u$. Similary, $A u=u=T u$. Hence, $u=A u=B u=S u=T u$. This represents that $u$ is the common fixed point of the mappings $A, B, S$ and $T$.

Uniqueness:

If possible, let $z(\neq u)$ be other common fixed point of the mappings, then by the condition (2) 


$$
\int_{0}^{d(u, z)} \phi(t) \mathrm{d} t=\int_{0}^{d(A u, B z)} \phi(t) \mathrm{d} t \leq k \int_{0}^{M(u, z)} \phi(t) \mathrm{d} t
$$

where

$$
\begin{aligned}
M(u, z) & =\max \{d(S z, A u) d(T u, S z), d(T u, A u), d(B z, S z), d(T u, B z)\} \\
& =\max \{d(z, u), d(u, z), d((u, u), d(z, z), d(u, z)\} \\
& =\max \{d(u, z), d(u, u), d(z, z)\}
\end{aligned}
$$

Since

$$
d(u, u) \leq 2 d(u, z) \text { and } d(z, z) \leq 2 d(z, u)
$$

So if $\max \{d(u, z), d(u, u), d(z, z)\}=d(u, z)$ or $d(u, u)$ or $d(z, z)$ we get the contradiction, since

$$
\int_{0}^{d(u, z)} \phi(t) \mathrm{d} t=\int_{0}^{d(A u, B z)} \phi(t) \mathrm{d} t \leq k \int_{0}^{M(u, z)} \phi(t) \mathrm{d} t \leq k \int_{0}^{d(u, z)} \phi(t) \mathrm{d} t
$$

or

$$
\int_{0}^{d(u, z)} \phi(t) \mathrm{d} t \leq 2 k \int_{0}^{d(u, z)} \phi(t) \mathrm{d} t
$$

or

$$
\int_{0}^{d(u, z)} \phi(t) \mathrm{d} t \leq 2 k \int_{0}^{d(u, z)} \phi(t) \mathrm{d} t
$$

Hence, $d(u, z)=0 \Rightarrow u=z$. This establishes the uniqueness of the common fixed point of four mappings.

Now we have the following corollaries:

If we take $T=S$ in Theorem (1) the we obtain the following corollary

Corollary 1 Let $(X, d)$ be a dislocated metric space. Let $A, B, S: X \rightarrow X$ satisfying the following conditions

$$
\begin{gathered}
A(X) \subseteq S(X) \quad \text { and } \quad B(X) \subseteq S(X) \\
\int_{0}^{d(A x, B y)} \phi(t) \mathrm{d} t \leq k \int_{0}^{M(x, y)} \phi(t) \mathrm{d} t, \quad k \in\left[0, \frac{1}{2}\right)
\end{gathered}
$$

where

$$
\phi: \mathbb{R}^{+} \rightarrow \mathbb{R}^{+}
$$

is a Lebesgue integrable mapping which is summable, non-negative and such that

$$
\begin{gathered}
\int_{0}^{\epsilon} \phi(t) \mathrm{d} t>0 \text { for each } \epsilon>0 \\
M(x, y)=\max \{d(S y, A x), d(S x, S y), d(S x, A x), d(B y, S y), d(S x, B y)\}
\end{gathered}
$$

1. The pairs $(A, S)$ or $(B, S)$ satisfy E. A. property.

2. The pairs $(A, S)$ and $(B, S)$ are weakly compatible.

if $S(X)$ is closed then

1) the maps $A$ and $S$ have a coincidence point

2) the maps $B$ and $S$ have a coincidence point

3 ) the maps $A, B$ and $S$ have an unique common fixed point.

If we take $B=A$ in Theorem (1) we obtain the following corollary.

Corollary 2 Let $(X, d)$ be a dislocated metric space. Let $A, S, T: X \rightarrow X$ satisfying the following conditions

$$
\begin{gathered}
A(X) \subseteq S(X) \quad \text { and } \quad A(X) \subseteq T(X) \\
\int_{0}^{d(A x, A y)} \phi(t) \mathrm{d} t \leq k \int_{0}^{M(x, y)} \phi(t) \mathrm{d} t, \quad k \in\left[0, \frac{1}{2}\right)
\end{gathered}
$$

where 


$$
\phi: \mathbb{R}^{+} \rightarrow \mathbb{R}^{+}
$$

is a Lebesgue integrable mapping which is summable, non-negative and such that

$$
\begin{gathered}
\int_{0}^{\epsilon} \phi(t) \mathrm{d} t>0 \quad \text { for each } \epsilon>0 \\
M(x, y)=\max \{d(S y, A x), d(T x, S y), d(T x, A x), d(A y, S y), d(T x, A y)\}
\end{gathered}
$$

1. The pairs $(A, T)$ or $(A, S)$ satisfy E. A. property.

2. The pairs $(A, T)$ and $(A, S)$ are weakly compatible.

if $T(X)$ is closed then

1) the maps $A$ and $T$ have a coincidence point.

2) the maps $A$ and $S$ have a coincidence point.

3) the maps $A, S$ and $T$ have an unique common fixed point.

If we take $T=S$ and $B=A$ in Theorem (1) then we obtain the following corollary

Corollary 3 Let $(X, d)$ be a dislocated metric space. Let $A, S: X \rightarrow X$ satisfying the following conditions

$$
\begin{gathered}
A(X) \subseteq S(X) \\
\int_{0}^{d(A x, A y)} \phi(t) \mathrm{d} t \leq k \int_{0}^{M(x, y)} \phi(t) \mathrm{d} t, \quad k \in\left[0, \frac{1}{2}\right)
\end{gathered}
$$

where

$$
\phi: \mathbb{R}^{+} \rightarrow \mathbb{R}^{+}
$$

is a Lebesgue integrable mapping which is summable, non-negative and such that

$$
\begin{gathered}
\int_{0}^{\epsilon} \phi(t) \mathrm{d} t>0 \text { for each } \epsilon>0 \\
M(x, y)=\max \{d(S y, A x), d(S x, S y), d(S x, A x), d(A y, S y), d(S x, A y)\}
\end{gathered}
$$

1. The pairs $(A, S)$ satisfy E. A. property.

2. The pair $(A, S)$ is weakly compatible.

if $S(X)$ is closed then maps $\mathrm{A}$ and $\mathrm{S}$ have a unique common fixed point.

If we put $S=T=I$ (Identity map) then we obtain the following corollary.

Corollary 4 Let $(X, d)$ be a dislocated metric space. Let $A, B, I: X \rightarrow X$ satisfying the following conditions

$$
\begin{gathered}
A(X), B(X) \subseteq X \\
\int_{0}^{d(A x, B y)} \phi(t) \mathrm{d} t \leq k \int_{0}^{M(x, y)} \phi(t) \mathrm{d} t, \quad k \in\left[0, \frac{1}{2}\right)
\end{gathered}
$$

where

$$
\phi: \mathbb{R}^{+} \rightarrow \mathbb{R}^{+}
$$

is a Lebesgue integrable mapping which is summable, non-negative and such that

$$
\begin{gathered}
\int_{0}^{\epsilon} \phi(t) \mathrm{d} t>0 \quad \text { for each } \epsilon>0 \\
M(x, y)=\max \{d(y, A x), d(x, y), d(x, A x), d(B y, y), d(x, B y)\}
\end{gathered}
$$

if the pair (A, B) satisfy E.A. property and are weakly compatible then the maps A and B have an unique common fixed point.

Remarks: Our result extends the result of [14].

Now we establish a fixed point theorem which generalize Theorem (2) of B. E. Rhoades [1].

Theorem 2 Let $(X, d)$ be a complete dislocated metric space, $k \in\left[0, \frac{1}{2}\right), f: X \rightarrow X$ be a mapping such 
that for each $x, y \in X$

$$
\int_{0}^{d(f x, f y)} \phi(t) \mathrm{d} t \leq k \int_{0}^{M(x, y)} \phi(t) \mathrm{d} t
$$

where

$$
M(x, y)=\max \left\{\frac{1}{2} d(y, f x), d(x, y), d(x, f x), d(y, f y), \frac{1}{2} d(x, f y)\right\}
$$

and

$$
\phi: \mathbb{R}^{+} \rightarrow \mathbb{R}^{+}
$$

is a lebesgue integrable mapping which is summable, non negative and such that

$$
\int_{0}^{\epsilon} \phi(t) \mathrm{d} t>0
$$

for each $\epsilon>0$, then $f$ has a unique fixed point $z \in X$, moreover for each $x \in X$

$$
\lim _{n \rightarrow \infty} f^{n} x=z
$$

Proof. Let $x \in X$ and define $y_{n}=f^{n} x, n \geq 1$, then from (18)

$$
\int_{0}^{d\left(y_{n}, y_{n+1}\right)} \phi(t) \mathrm{d} t \leq k \int_{0}^{M\left(y_{n-1}, y_{n}\right)} \phi(t) \mathrm{d} t
$$

now by (19)

$$
\begin{aligned}
M\left(y_{n-1}, y_{n}\right) & =\max \left\{\frac{1}{2} d\left(y_{n}, f y_{n-1}\right), d\left(y_{n-1}, y_{n}\right), d\left(y_{n-1}, f y_{n-1}\right), d\left(y_{n}, f y_{n}\right), \frac{1}{2} d\left(y_{n-1}, f y_{n}\right)\right\} \\
& =\max \left\{\frac{1}{2} d\left(y_{n}, y_{n}\right), d\left(y_{n-1}, y_{n}\right), d\left(y_{n-1}, y_{n}\right), d\left(y_{n}, y_{n+1}\right), \frac{1}{2} d\left(y_{n-1}, y_{n+1}\right)\right\} \\
& =\max \left\{\frac{1}{2} d\left(y_{n}, y_{n}\right), d\left(y_{n-1}, y_{n}\right), d\left(y_{n}, y_{n+1}\right), \frac{1}{2} d\left(y_{n-1}, y_{n+1}\right)\right\}
\end{aligned}
$$

But,

$$
\begin{aligned}
& \frac{1}{2} d\left(y_{n-1}, y_{n+1}\right) \leq \frac{1}{2}\left[d\left(y_{n-1}, y_{n}\right)+d\left(y_{n}, y_{n+1}\right)\right] \\
& =\max \left\{d\left(y_{n-1}, y_{n}\right), d\left(y_{n}, y_{n+1}\right)\right\}
\end{aligned}
$$

and similarly we can obtain, $\frac{1}{2} d\left(y_{n}, y_{n}\right) \leq d\left(y_{n}, y_{n+1}\right)$

Hence $M\left(y_{n-1}, y_{n}\right)=\max \left\{d\left(y_{n-1}, y_{n}\right), d\left(y_{n}, y_{n+1}\right)\right\}$

Therefore by (21)

$$
\begin{aligned}
\int_{0}^{d\left(f y_{n-1}, f y_{n}\right)} \phi(t) \mathrm{d} t & =\int_{0}^{d\left(y_{n}, y_{n+1}\right)} \phi(t) \mathrm{d} t \leq k \int_{0}^{\max \left\{d\left(y_{n-1}, y_{n}\right), d\left(y_{n}, y_{n+1}\right)\right\}} \phi(t) \mathrm{d} t \\
& =k \max \left\{\int_{0}^{d\left(y_{n-1}, y_{n}\right)} \phi(t) \mathrm{d} t, \int_{0}^{d\left(y_{n}, y_{n+1}\right)} \phi(t) \mathrm{d} t\right\} \\
& =k \int_{0}^{d\left(y_{n-1}, y_{n}\right)} \phi(t) \mathrm{d} t
\end{aligned}
$$

Similarly we can obtain,

$$
\int_{0}^{d\left(y_{n-1}, y_{n}\right)} \phi(t) \mathrm{d} t \leq k \int_{0}^{d\left(y_{n-2}, y_{n-1}\right)} \phi(t) \mathrm{d} t
$$

Hence

$$
\int_{0}^{d\left(y_{n}, y_{n+1}\right)} \phi(t) \mathrm{d} t \leq k \int_{0}^{d\left(y_{n-1}, y_{n}\right)} \phi(t) \mathrm{d} t \leq k^{2} \int_{0}^{d\left(y_{n-2}, y_{n-1}\right)} \phi(t) \mathrm{d} t \leq \cdots \leq k^{n} \int_{0}^{d\left(y_{0}, y_{1}\right)} \phi(t) \mathrm{d} t
$$


Now taking limit as $n \rightarrow \infty$ we get

$$
\lim _{n \rightarrow \infty} \int_{0}^{d\left(y_{n}, y_{n+1}\right)} \phi(t) \mathrm{d} t=0
$$

by $(20)$

$$
\lim _{n \rightarrow \infty} d\left(y_{n}, y_{n+1}\right)=0
$$

Now we claim that $\left\{y_{n}\right\}$ is a Cauchy sequence.

If possible let $\left\{y_{n}\right\}$ is not a Cauchy sequence, then there exists a real number $\epsilon>0$ and subsequences $q_{i}$ and $p_{i}$ such that $p_{i}<q_{i}<p_{i+1}$ and

$$
d\left(y_{p_{i}}, y_{q_{i}}\right) \geq \varepsilon \text { and } d\left(y_{p_{i}}, y_{q_{i-1}}\right)<\varepsilon
$$

Using (19) we have,

$$
M\left(y_{p_{i-1}}, y_{q_{i-1}}\right)=\max \left\{\frac{1}{2} d\left(y_{q_{i-1}}, y_{p_{i}}\right), d\left(y_{p_{i-1}}, y_{q_{i-1}}\right), d\left(y_{p_{i-1}}, y_{p_{i}}\right), d\left(y_{q_{i-1}}, y_{q_{i}}\right), \frac{1}{2} d\left(y_{p_{i-1}}, y_{q_{i}}\right)\right\}
$$

Now using (22)

$$
\lim _{i \rightarrow \infty} \int_{0}^{d\left(y_{p_{i-1}}, y_{p_{i}}\right)} \phi(t) \mathrm{d} t=\lim _{i \rightarrow \infty} \int_{0}^{d\left(y_{q_{i-1}}, y_{q_{i}}\right)} \phi(t) \mathrm{d} t=0
$$

Since by triangle inequality and (23)

$$
d\left(y_{p_{i-1}}, y_{q_{i-1}}\right) \leq d\left(y_{p_{i-1}}, y_{p_{i}}\right)+d\left(y_{p_{i}}, y_{q_{i-1}}\right)<d\left(y_{p_{i-1}}, y_{p_{i}}\right)+\varepsilon
$$

Hence

$$
\lim _{i \rightarrow \infty} \int_{0}^{d\left(y_{p_{i-1}}, v_{i-1}\right)} \phi(t) \mathrm{d} t \leq \lim _{i \rightarrow \infty} \int_{0}^{\varepsilon} \phi(t) \mathrm{d} t
$$

and

$$
\begin{gathered}
\frac{1}{2} d\left(y_{p_{i-1}}, y_{q_{i}}\right) \leq \frac{1}{2}\left[d\left(y_{p_{i-1}}, y_{p_{i}}\right)+d\left(y_{p_{i}}, y_{q_{i}}\right)\right] \leq \frac{1}{2} d\left(y_{p_{i-1}}, y_{p_{i}}\right)+\frac{\varepsilon}{2} \\
\lim _{n \rightarrow \infty} \int_{0}^{\frac{1}{2} d\left(y_{p_{i-1}}, y_{q_{i}}\right)} \phi(t) \mathrm{d} t \leq \int_{0}^{\frac{\varepsilon}{2}} \phi(t) \mathrm{d} t
\end{gathered}
$$

Similarly

$$
\lim _{n \rightarrow \infty} \int_{0}^{\frac{1}{2} d\left(y_{q_{i-1}}, y_{p_{i}}\right)} \phi(t) \mathrm{d} t \leq \int_{0}^{\frac{\varepsilon}{2}} \phi(t) \mathrm{d} t
$$

Hence, from (20), (23), (24), (25), (26), (27) and (28)

$$
\int_{0}^{\varepsilon} \phi(t) \mathrm{d} t \leq \int_{0}^{d\left(y_{p_{i}}, y_{q_{i}}\right)} \phi(t) \mathrm{d} t \leq k \int_{0}^{M\left(y_{p_{i-1}}, y_{q_{i-1}}\right)} \phi(t) \mathrm{d} t \leq k \int_{0}^{\varepsilon} \phi(t) \mathrm{d} t
$$

which is a contradiction. Hence $\left\{y_{n}\right\}$ is a Cauchy sequence. Hence there exists a point $z \in X$ such that the sequence $\left\{y_{n}\right\}$ and its subsequences converge to $z$.

From the condition (18)

$$
\begin{aligned}
& \int_{0}^{d\left(f z, y_{n+1}\right)} \phi(t) \mathrm{d} t \leq k \int_{0}^{M\left(z, y_{n}\right)} \phi(t) \mathrm{d} t \\
& =k \max \left\{\int_{0}^{\frac{1}{2} d\left(y_{n}, f\right)} \phi(t) \mathrm{d} t, \int_{0}^{d\left(z, y_{n}\right)} \phi(t) \mathrm{d} t, \int_{0}^{d(z, f z)} \phi(t) \mathrm{d} t, \int_{0}^{d\left(y_{n}, y_{n}\right)} \phi(t) \mathrm{d} t, \int_{0}^{\frac{1}{2} d\left(z, f y_{n}\right)} \phi(t) \mathrm{d} t\right\}
\end{aligned}
$$

Now taking limit as $n \rightarrow \infty$ we obtain

$$
\int_{0}^{d(f z, z)} \phi(t) \mathrm{d} t \leq k \int_{0}^{d(z, f z)} \phi(t) \mathrm{d} t
$$


which implies

$$
\int_{0}^{d(f, z)} \phi(t) \mathrm{d} t=0
$$

So from the relation (20) we obtain $d(f z, z)=0 \Rightarrow f z=z$

\section{Uniqueness:}

Let $z$ and $w$ two fixed point fixed points of the function $f$.

Applying condition (19) we obtain

$$
\begin{aligned}
& \int_{0}^{d(z, w)} \phi(t) \mathrm{d} t=\int_{0}^{d(f z, f w)} \phi(t) \mathrm{d} t \leq k \int_{0}^{M(z, w)} \phi(t) \mathrm{d} t \\
& =k \max \left\{\int_{0}^{\frac{1}{2} d(w, z)} \phi(t) \mathrm{d} t, \int_{0}^{d(z, w)} \phi(t) \mathrm{d} t, \int_{0}^{d(z, z)} \phi(t) \mathrm{d} t, \int_{0}^{d(w, w)} \phi(t) \mathrm{d} t, \int_{0}^{\frac{1}{2} d(z, w)} \phi(t) \mathrm{d} t\right\} \\
& =k \max \left\{\int_{0}^{d(z, w)} \phi(t) \mathrm{d} t, \int_{0}^{d(z, z)} \phi(t) \mathrm{d} t, \int_{0}^{d(w, w)} \phi(t) \mathrm{d} t\right\}
\end{aligned}
$$

If maximum of the given expression in the set is $\int_{0}^{d(z, z)} \phi(t) \mathrm{d} t$ then

$$
\int_{0}^{d(z, w)} \phi(t) \mathrm{d} t \leq k \int_{0}^{d(z, z)} \phi(t) \mathrm{d} t \leq k \int_{0}^{2 d(z, w)} \phi(t) \mathrm{d} t=2 k \int_{0}^{d(z, w)} \phi(t) \mathrm{d} t
$$

which is a contradiction, since $k \in\left[0, \frac{1}{2}\right)$. Similarly for other cases also we get the contradiction. Hence $z=w$. This completes the proof of the theorem.

\section{References}

[1] Rhoades, B.E. (2003) Two Fixed Point Theorems for Mappings Satisfying a General Contractive Condition of Integral Type. International Journal of Mathematics and Mathematical Sciences, 63, 4007-4013. http://dx.doi.org/10.1155/S0161171203208024

[2] Matthews, S.G. (1986) Metric Domains for Completeness. Technical Report 76, PhD Thesis, Department of Computer Science, University of Warwick, Coventry.

[3] Hitzler, P. and Seda, A.K. (2000) Dislocated Topologies. Journal of Electrical Engineering, 51, 3-7.

[4] Kumari, P.S., Zoto, K. and Panthi, D. (2015) D-Neighborhood System and Generalized F-Contraction in Dislocated Metric Space. Springer Plus, 4, 1-10. http://dx.doi.org/10.1186/s40064-015-1095-3

[5] Kumari, P.S. (2012) Common Fixed Point Theorems on Weakly Compatible Maps on Dislocated Metric Spaces. Mathematical Sciences, 6, 71. http://dx.doi.org/10.1186/2251-7456-6-71

[6] Kumari, P.S. and Panthi, D. (2015) Cyclic Contractions and Fixed Point Theorems on Various Generating Spaces. Fixed Point Theory and Applications, 2015, 153. http://dx.doi.org/10.1186/s13663-015-0403-5

[7] Kumari, P.S., Ramana, C.V., Zoto, K. and Panthi, D. (2015) Fixed Point Theorems and Generalizations of Dislocated Metric Spaces. Indian Journal of Science and Technology, 8, 154-158. http://dx.doi.org/10.17485/ijst/2015/v8iS3/62247

[8] Panthi, D. (2015) Common Fixed Point Theorems for Compatible Mappings in Dislocated Metric Space. International Journal of Mathematical Analysis, 9, 2235-2242. http://dx.doi.org/10.12988/ijma.2015.57177

[9] Panthi, D. and Jha, K. (2012) A Common Fixed Point of Weakly Compatible Mappings in Dislocated Metric Space. Kathmandu University Journal of Science, Engineering and Technology, 8, 25-30.

[10] Sarma, I.R., Rao, J.M., Kumari, P.S. and Panthi, D. (2014) Convergence Axioms on Dislocated Symmetric Spaces. Abstract and Applied Analysis, 2014, Article ID: 745031. http://dx.doi.org/10.1155/2014/745031

[11] Branciari, A. (2002) A Fixed Point Theorem for Mappings Satisfying a General Contractive Condition of Integral Type. International Journal of Mathematics and Mathematical Sciences, 29, 531-536. http://dx.doi.org/10.1155/S0161171202007524

[12] Jungck, G. and Rhoades, B.E. (1998) Fixed Points for Set Valued Functions without Continuity. Indian Journal of Pure and Applied Mathematics, 29, 227-238.

[13] Amri, M. and El Moutawakil, D. (2002) Some New Common Fixed Point Theorems under Strict Contractive Condi- 
tions. Journal of Mathematical Analysis and Applications, 270, 181-188. http://dx.doi.org/10.1016/S0022-247X(02)00059-8

[14] Kumar, J. (2013) Common Fixed Point Theorems of Weakly Compatible Maps Satisfying (E.A) and (CLR) Property. Indian Journal of Pure and Applied Mathematics, 88, 363-376. http://dx.doi.org/10.12732/ijpam.v88i3.4 